\title{
Postoperative patient controlled analgesia in elderly Koreans: epidural versus intravenous administration
}

\author{
Shin-Hyung Kim ${ }^{1}$, Yang-Sik Shin ${ }^{1,2}$, Young-Jun $\mathrm{Oh}^{1,2}$, In-Hye Park ${ }^{1}$, and Yong-Seon Choi ${ }^{1,2}$ \\ ${ }^{1}$ Department of Anesthesiology and Pain Medicine, ${ }^{2}$ Anesthesia and Pain Research Institute, Yonsei University College of Medicine, \\ Seoul, Korea
}

Patient-controlled analgesia (PCA) techniques are widely used for postoperative pain control, but the efficacy and safety of these modalities have been poorly evaluated in elderly Koreans. Therefore, we compared the effect of intravenous and epidural PCA on the postoperative pain and adverse events in elderly patients undergoing major abdominal surgery.

Patients (> 65 years) who received intravenous PCA (IV PCA) or epidural PCA (PCEA) after major abdominal surgery under general anesthesia between March 2011 and March 2012 were included in the study. In the IV PCA group, patients received IV PCA using fentanyl (14-18 $\mu \mathrm{g} / \mathrm{kg})$ plus $8 \mathrm{mg}$ of ondansetron or $0.3 \mathrm{mg}$ of ramosetron, which was programmed to deliver $2 \mathrm{ml} / \mathrm{h}$ as a background infusion, and $0.5 \mathrm{ml}$ per demand with a 15 min lockout. In the PCEA group, patients received PCEA using fentanyl $(2-5 \mu \mathrm{g} / \mathrm{ml})$ plus ropivacaine $(0.10-0.20$ $\%$ ), which was programmed to deliver $5 \mathrm{ml} / \mathrm{h}$ as a background infusion and $2 \mathrm{ml}$ per demand, with a $15 \mathrm{~min}$ lockout. Epidural catheters were inserted before surgery at a vertebral level corresponding to the dermatomal level of the surgical incision. For patients presenting with difficult thoracic epidural catheterization requirements, epidural catheters were inserted near the site of surgical incision. At the end of surgery, IV PCA or PCEA was initiated. Two PCA nurse practitioners monitored patients in the postanesthesia care unit (PACU) and at 1-6, 6-12, 12-18, 18-24 and $24-48 \mathrm{hr}$ intervals after surgery, inquiring about the occurrence of adverse events, the need for rescue IV analgesics (15-30 $\mathrm{mg}$ of ketolorac, 25-50 mg of tramadol or $25 \mathrm{mg}$ of meperidine), and pain intensity scores.
A total of 1024 patients were included in the study; 754 patients in the IV PCA group and 270 patients in the PCEA group. Patient characteristics, anesthesia and operative data were similar between the two groups except that female gender and ASA physical status $\geq$ III were more common in the IV PCA group. In the PCEA group, $72 \%$ of patients underwent laparotomy through an upper-mid abdominal incision. Patients who underwent laparoscopic or robot-assisted surgery mostly were mainly treated with IV PCA (95\%). While pain intensity was similar between the two groups in the PACU, it was significantly higher in the PCEA group from 1 to $48 \mathrm{~h}$ after surgery. The need for rescue analgesics was less in the IV PCA group compared to the PCEA group during 6 to $48 \mathrm{~h}$ following surgery. In subgroup analysis according to surgical site, pain intensity was similar throughout the study period between the two routes of administration among patients who underwent upper-mid laparotomy. However, in patients who underwent mid-lower laparotomy and laparoscopic or robot-assisted surgery, pain intensity was higher in the PCEA group compared to the IV PCA group during the first 1 to $48 \mathrm{~h}$ period and in the PACU to $48 \mathrm{~h}$ period, respectively (Fig. 1). There were no significant differences in adverse events including incidence of post-operative nausea and vomiting, headache and dizziness, urinary retention, or sedation between the two groups.

Epidural analgesia has been reported to be superior to intravenous analgesia with respect to postoperative pain relief, bowel recovery, and patient satisfaction [1,2]; epidural analgesia has also been reported to be similar to intravenous analgesia in

Corresponding author: Yong-Seon Choi, M.D., Department of Anesthesiology and Pain Medicine, Yonsei University College of Medicine, 50, Yonsei-ro, Seodaemun-gu, Seoul 120-752, Korea. Tel: 82-2-2227-4576, Fax: 82-2-364-2951, E-mail: yschoi@yuhs.ac

(c) This is an open-access article distributed under the terms of the Creative Commons Attribution Non-Commercial License (http:// creativecommons.org/licenses/by-nc/3.0/), which permits unrestricted non-commercial use, distribution, and reproduction in any medium, provided the original work is properly cited. 
A
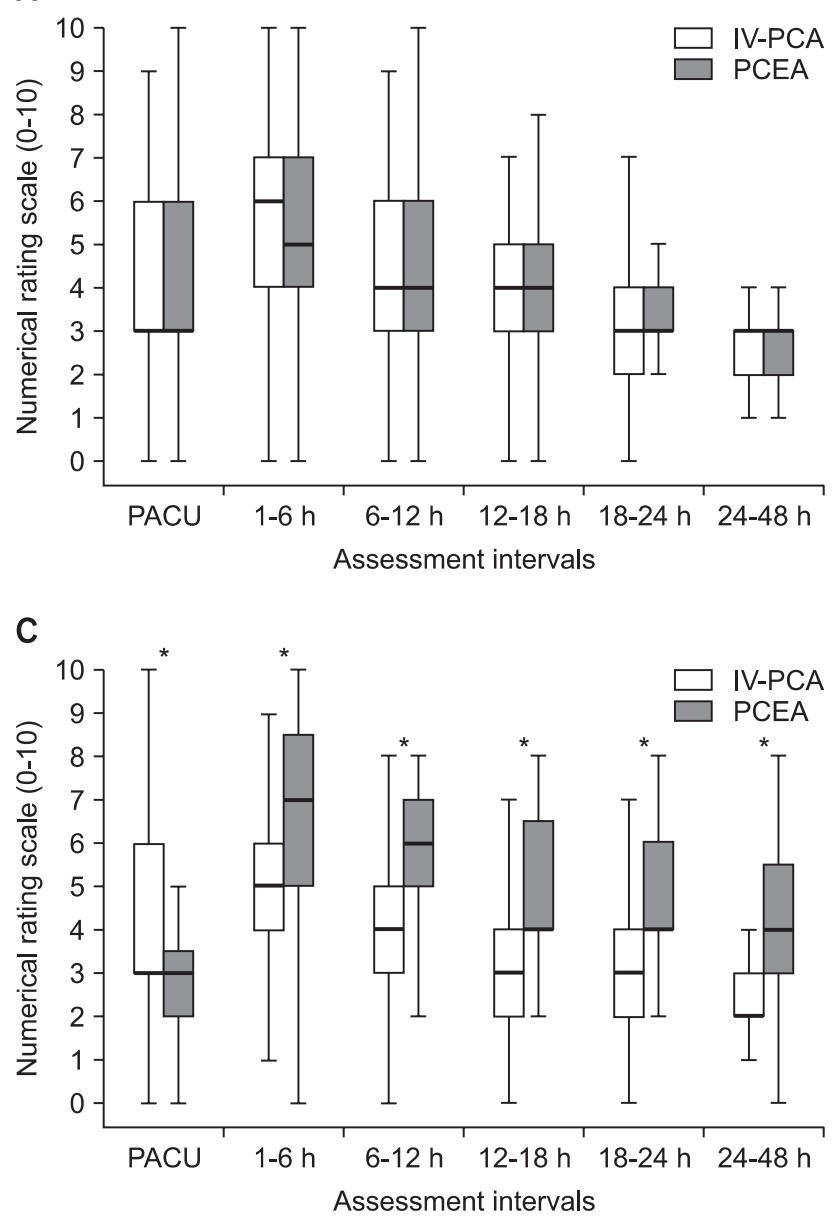

terms of the incidence of major morbidity and duration of hospital stay after major abdominal surgery [3]. To the contrary, our results demonstrated that the IV PCA provided better analgesia than PCEA during the first 1-48 $\mathrm{h}$ period after major abdominal surgery in elderly patients. It is notable, however, that in subgroup analysis by surgical site, postoperative pain relief was similar between the two PCA routes of administration in patients who underwent upper-mid laparotomy. These results have several possible explanations. First, fixed PCA devices are commonly used in clinical settings in Korea, but most previous studies evaluating epidural analgesia used adjustable PCA device settings to allow for more flexibility in the timing and dose delivered via the epidural route [1]. In this study, however, postoperative pain intensity was comparable between the IV PCA and PCEA techniques only in the PACU because we administered additional local anesthetics via epidural catheter at the discretion of the attending anesthesiologists during surgery and/or in the PACU. Second, we found that the benefits of postoperative epidural analgesia could be optimized when the epidural catheter was placed in a location corresponding to the dermatome
B

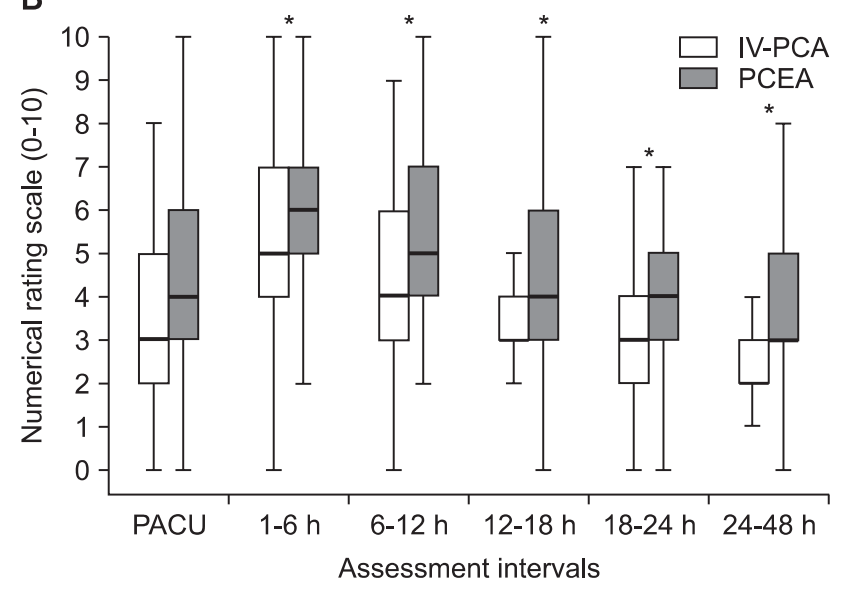

Fig. 1. Postoperative pain intensity according to site of surgery. (A) laparotomy using upper-mid abdominal incision (B) laparotomy using mid-lower abdominal incision, (C) laparoscopic or robot-assisted abdominal surgery. PACU: post-anesthesia care unit. $* \mathrm{P}<0.05$ between two groups.

of the surgical incision. In our study, $93 \%$ of patients who had an upper-mid laparotomy and $78 \%$ of patients who had a midlower laparotomy subgroup received epidural catheterization at thoracic levels. These results were consistent with analgesic efficacy, which was comparable with PCEA and IV PCA techniques only in upper-mid laparotomy subgroup, likely due to difficulty of placement at thoracic levels. Placement of epidural catheters may be difficult in the elderly because of degenerative anatomical changes, obesity, or poor cooperation and narrow thoracic vertebrae spaces [4]. Third, laparoscopic or robot-assisted abdominal surgery is less invasive than conventional laparotomy, but postoperative analgesia requires a relatively broad sensory blockade and $\mathrm{CO}_{2}$ pneumoperitoneum is associated with a high incidence of musculoskeletal pain such as shoulder pain [5]. Thus, IV PCA might have advantages over PCEA in elderly patients undergoing this particular surgical technique.

This study is not without limitations. Because it was observational, we used a real-world clinical practice model in which attending physicians decided on the placement of epidural catheters and the prescription of the PCA regimen according to 
institutional guidelines. The differences in gender, ASA physical status and variations of surgery between IV PCA and PCEA groups might have affected our results. Therefore, further studies will be needed that take these limitations into consideration; a more controlled study of PCA in Korea is necessary.

In conclusion, we found that PCEA was not superior to IV PCA for analgesic efficacy after major abdominal surgery in elderly patients in whom proper epidural catheter positioning was not confirmed. Careful placement of epidural catheters and sustainable PCEA monitoring is needed to achieve maximal benefits of PCEA for elderly patients.

\section{Acknowledgments}

We wish to thank PCA nurse practitioner Won-Hee Baek, R.N. and Sun-Young Oh, R.N. for their expert assistance in collecting data.

\section{References}

1. Mann C, Pouzeratte Y, Boccara G, Peccoux C, Vergne C, Brunat G, et al. Comparison of intravenous or epidural patient-controlled analgesia in the elderly after major abdominal surgery. Anesthesiology 2000; 92: 433-41.

2. Mann C, Pouzeratte Y, Eledjam J. Postoperative patient-controlled analgesia in the elderly: risks and benefits of epidural versus intravenous administration. Drugs Aging 2003; 20: 337-45.

3. Peyton PJ, Myles PS, Silbert BS, Rigg JA, Jamrozik K, Parsons R. Perioperative epidural analgesia and outcome after major abdominal surgery in high-risk patients. Anesth Analg 2003; 96: 548-54.

4. Adachi YU, Sanjo Y, Sato S. The epidural space is deeper in elderly and obese patients in the Japanese population. Acta Anaesthesiol Scand 2007; 51: 731-5.

5. Nishikawa K, Kimura S, Shimodate Y, Igarashi M, Namiki A. A comparison of intravenous-based and epidural-based techniques for anesthesia and postoperative analgesia in elderly patients undergoing laparoscopic cholecystectomy. J Anesth 2007; 21: 1-6. 